\title{
Heuristic Algorithm Based on MAXDCP and GEOCP in Grid Network
}

\author{
Shaohui Ma and Xiangqian Chen \\ Department of Computer Science and Technology, Henan Mechanic and \\ Electrical Engineering College, Henan Xinxiang, China \\ E-mail:xxhnshm@yeah.net
}

\begin{abstract}
In order to solve issues of grid network as a broadband wireless access network, such as high data transmission rate and long-distance signal coverage and so on, this paper proposes two heuristic algorithms with $O(\mathrm{~m} 3)$ time complexity, based on the user neighbors' information and location information. The algorithm will meet transferring the minimum relay deployment problem required by users into the minimum clique partition problem of adjacency graph and gives specific algorithm steps. Experimental results show that: the proposed algorithm ensures network performance and reduces network cost.
\end{abstract}

Keywords: Data Transmission, Station, Inspire, Approximation

\section{Introduction}

WIMAX Grid network as a broadband wireless access network, has the characteristics such as low cost, high data transfer rates and long-distance signal coverage. However, when the base station's transmission power is constant, the noise ratio of the received signal will exponentially decrease as the distance from the base station increases, the obtained data transfer rate decreases [1-3]; If WIMAX cell radius is too large, since the wireless signal has propagational characteristics like sight transmission and attenuation, users in the shaded area in the building and at the edge of base station coverage usually get low signal noise ratio. Therefore, the user terminal access to mobile wireless broadband's data rate is limited, affecting the quality and development of mobile Internet application and service [4].

In order to solve the above problem, IEEE802.16j standard combines multi-hop relay technology with the Grid network mode, and adopts tree topology. The WIMAX network increases relays in the propagation path, forming a access link (Access Link) from users to the relay and the relay access link (Relay Link) from users to the base station, which has advantages with low cost, high bandwidth, wide coverage, and it is a new direction in technical aspects of the 802.16 standard [5-7].

Deploying relays to improve network service quality and reducing network cost has become one of the main issues of theory study on WIMAX Grid [8-10]. Wherein, network service quality is presented by the transmission rate of users' access to the link, and when the base station's transmission power and the number of channels are fixed, the rate is directly dependent on the distance between the users to the relay. Zhang etc., proposed that when the distance requirements from the user to the relay is known, the deploy the least relay to ensure the communication between the user and the station (Distance-Aware Relay Placement, DARP), the DARP problems were resolved into two sub-problems:(1) Firstly, deployed minimum relay to ensure the user's requirements in access link; (2) and then deploy least relay to ensure communication of relay previously deployed and the base station. Which, DARP'S optimized key step is reducing the number of deployed relay of the sub-problem (1), therefore, Zhang proposed 
approximation algorithm MIS and HS. However, MIS only considered to change problem into the general graphic information, regarded the user's distribution, resulting in deploying redundant relay; HS'S approximation is $(1+\beta)$, but the time complexity is $O\left(m n_{0}(\beta-3)\right)$, wherein $\mathrm{m}$ is the number of users, $\mathrm{n}$ is the number of the relay, this means that in order to get a better approximation $(\beta \rightarrow 0)$, HS algorithm's time complexity is too high and is not suitable for large-scale practical application [11-15].

This paper proposes two heuristic algorithms with $O\left(m_{3}\right)$ time complexity, based on the user neighbor's information and location information. The algorithm will meet transferring the minimum relay deployment problem required by users' requirements into the smallest group partitioning problem of adjacency graph and gives specific algorithm steps. Simulation results show that the algorithm can meet all the user's distance requirements with fewer relay and lower time complexity and maintain connectivity with the base station.

This paper mainly made expanding and innovative work in the following areas:

(1)In order to solve issues of WIMAX Grid network as a broadband wireless access network, such as high data transmission rate and long-distance signal coverage and so on, this paper proposes two heuristic algorithms with $\mathrm{O}$ ( $\mathrm{m} 3$ ) time complexity, based on the user neighbors' information and location information. This paper studies the WIMAX Grid network's least relay deployment to meet the user's reliable coverage LORC, firstly proves that LORC issue is equivalent to solving problems of least group partitioning in the user adjacency graph, the problem is NP-complete. Then, based on user neighbor information and user location information, proposes greedy heuristics MAXDCP and GEOCP, which will meet the condition changing the least relay deployment problem required by users' distance requirement into the smallest group partitioning problem in adjacency graph.

(2) In order to further validate the proposed heuristic algorithm MAXDCP and GEOCP'S correctness and validity of the simulation experiments, compared to the latest algorithm MIS, with same time complexity, the number of deployed relay of MAXDCP reduces $23.8 \%$ average, GEOCP has an average reduction of $35 \%$; compared with the existing HS of PTAS algorithm, the number of GEOCP'S deployed relay average reduces $18.5 \%$, in the same time with lower time complexity. MAXDCP and GEOCP ensure the network performance and reduce network costs well. The simulation results show that: the proposed algorithm guarantees the user's distance requirements, compared with existing algorithms; it has advantages such as with fewer deployed relay and lower time complexity.

\section{Model and Problem Description}

In this paper, WIMAX Grid network's static multi-hop relay deployment issues are studied, the ultimate optimizing goal is to ensure users' transmission rate. Because when the number of channels is fixed, the user receiving data transmission rate is proportional to reception channel capacity, while in the WIMAX network with massive long signal communication, generally adopts Two-ray Ground Loss model, according to Shannon's theorem, channel capacity $D$ is expressed as:

$$
D=j \log \left(1+T R E_{i}\right)
$$

Wherein, $j$ is the channel bandwidth, $T R E_{i}$ is the signal noise ratio, according to the Two-ray Ground Loss model,

$$
T R E_{i}=\frac{G_{i} D_{i} D_{y} k_{i}^{2} k_{i}^{s} d^{\alpha}}{R_{0}}
$$


Where, $\mathrm{Pt}$ is the transmission power, $D_{i} / D_{y}$ and $k_{i} / k_{y} \mathrm{~h}$, respectively, for transmitting / receiving antenna gain and height, $R_{0}$ is the thermal noise, usually, these parameters are constants. Thus, by the formula (1), (2), when the transmission power is constant, the channel capacity is decided by the physical distance between reception side and the transmission side. Based on the above analysis, the user's data rate depends on the distance from the user to the relay, inverse relationship between the two. That is, assuming in order to ensure the transmission rate of the user a minimum $k$, the required maximum physical distance to relay is $D$ meters, then the "relay deployment for ensuring the transmission rate of the user " problem is equivalent to the "in the user-centric area with d radius, at least deploy one relay" problem.

Therefore, when the base station transmit power and number of channels reaches a certain level, "to ensure transmission rate of users " is equivalent to" to ensure distance requirements of users to the relay".

\section{A. Network Model}

$R$ users $w=\left\{w_{1}, w_{2}, \ldots w_{R}\right\}$ randomly distributed in an square area A with $l \times l_{R_{2}}$, and the corresponding distance requirements to each user's transmission rate is $D=\left\{D_{1}, D_{2}, \ldots D_{R}\right\}$. Within A, there is one base station BS. Other assumptions about the network model as follows.

(1) The user is stationary. In actual scene, this assumption corresponds to gas stations, airports, supermarkets and other fixed place's placed terminals. For mobile users, such as mobile phones users and users of notebook which with WIMAX network, they can exchange data with the base station through indoor or outdoor stationary user equipment (fixed subscriber antennas) and the multi-hop transmission of the relay.

(2) Once relay placed, no longer moving. IEEE 802.16j defines three types of repeaters, they are fixed relay (Fix Relay Station, FRS), patrol relay (Nomadic Relay Station, NRS) and the mobile relay (Mobile Relay Station, MRS), This paper study static networks' deployment issues, so uses fixed relay, that is once relay is placed, the location and number will be no longer changed.

(3) Based on the "user-to-relay and relay-to-base" double-layer relay model, as shown in Figure 1, the relay deployment theory in multi-hop relay WIMAX network is widely used in research, this paper studies "User-to -relay "relay deployment issues of access to link .

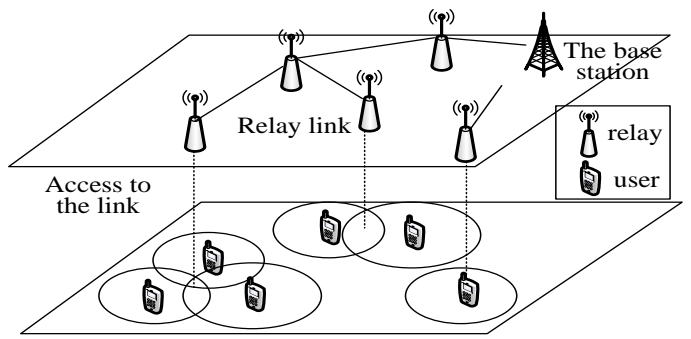

Figure 1. "User-To-Relay - Relay-To -Base Station" Double Layer Relay Model

(4) In this paper, we focus on how to deploy the fewest hops to ensure that users can communicate with the base station through multi-hop relay by the minimum required rate. As for one relay in charge of multiple users' access, the channel multiplexing and user scheduling to solve throughput problems although is also affected by the relay deployment, but it is not in the scope of this paper's consideration.

\section{B. Related Definitions}




\section{Definition 1: Distance Circle}

For user $m_{i}$, its user distance requirement is $c_{i}$, the distance circle of user $m_{i}$ takes the location of $m_{i}$ as the center, the perfect circle with $g_{i}$ radius is denoted by $e_{i}$.

\section{Definition 2: User Adjacency Graph}

User adjacency graph is a graph $g(T, e)$, where $T$ is the set of vertexes, there is one-to-one correspondence between each vertex and the users in $\mathrm{S}$, unless otherwise specified below, a vertex represents a user; $\mathrm{E}$ is the set of edges, for vertexes $T_{i}$ and $T_{j}$ in $T$, there is an edge $g_{i j}\left(T_{i}, T_{j}\right)$ in $\mathrm{E}$ if and only if the distance circle between $T_{i}$ and $T_{j}$ has non-empty intersection. In particular, when $g(T, e)$ has only one vertex $T_{i}$, that is, $\mathrm{G}$ has isolated pole, let $T=\left\{e_{12}\left(T_{i}, T_{j}\right)\right\}$.

\section{Definition 3: Reliable Coverage}

If the distance circle $e_{i}$ of $T_{i}$ has at least one repeater or base station, then vi is called reliable coverage.

\section{Definition 4: Complete Graph}

Given a graph $g(T, e)$, if there is edge between any pair of vertexes, then we say this graph is a complete graph.

\section{Definition 5: Group}

For Figure $g(T, e), \exists \vee, \subseteq \vee$, if the exported $\operatorname{sub}_{\text {graph }} g\left(T^{\prime}, e^{\prime}\right)$ from the vertex set $T^{\prime}$ is a complete graph, then call $T$ ' as the group in graph $T$. If a group is not contained in any other group, which means it is not one of subset of other groups, then we call this group as the maximal clique of graph $T$ (maximal clique); Maximal clique with most vertexes becomes the largest group of $T$ (maximum clique).

\section{Description}

The least relay deployment which ensures transfer rate issue (Distance-Aware Relay Placement in WIMAX Grid Networks, DARP): In a WIMAX Grid network, given one base station BS and the user set $w=\left\{w_{1}, w_{2}, \ldots w_{R}\right\}$, the distance set corresponding to the user data rate requirement is $D=\left\{D_{1}, D_{2}, \ldots D_{R}\right\}$, deploy minimum relay $R_{w}$, makes that

(1) For $\forall w_{i} \subseteq w$, in the area with $w_{i}$ as the center and with a radius $d_{i}$, there should be at least one repeater or base station, that is in the user-to-relay layer, deploying least relay to guarantee reliable coverage for each user (Lower -tier Relay Coverage, LORC);

(2) Each relay RS can communicate with the base station with the required data rate, $i, e$. in the "relay-to-base station" layer, deploy the least relay to ensure relay can communicate with the base station with the desired rate.

Among them, the second sub-problem takes the deployed relay of LORC as the leaf node, and the base station as the root, to build side-constrained Euclidean Steiner tree (MUST), so the optimization key of DARP is the solving of the LORC problem.

\section{Heuristic Algorithm}

First, changes LORC problem into the smallest group partitioning problem in user adjacency graph (Minimum Clique Partition), and then proposes two heuristic algorithms, finally analyzes the algorithm's time complexity. 
Theorem 1. In non-empty user adjacency graph $g(T, e)$, let a vertex's minimum distance requirement as $d_{\min }, g\left(T^{\prime}, e^{\prime}\right)$ is one of the groups in $g(T, e),|T|$ is the number of vertexes within the group.

(1) If $0 \prec|T| \prec 3$, then only one relay can ensure the reliable coverage of all vertexes in the group.

(2) If $|T| \geq 3$ and the distance between any two vertexes in the group is not more than $|T| \geq 3$, then only one relay can ensure the reliable coverage of all vertexes in the group.

Proof. Let $g\left(T^{\prime}, e^{\prime}\right)$ as a group in the user adjacency $\operatorname{graph} g(T, e)$, if the distance between any two vertexes in the group is not more than $d_{\min }$, $\exists e_{i, j} \in E^{\prime}, S_{i} \cap S_{j} \neq \varnothing, T_{i} T_{j} \leq \sqrt{3} d_{\text {min }}$, so

$$
\bigcap_{s_{i} \in s^{\prime}} S_{j} \neq \varnothing
$$

(1) When $\left|T^{\prime}\right|=1, i, e$ in group $\mathrm{G}^{\prime}$, there is only one vertex, simply place a repeater anywhere within this vertex's distance circle can ensure reliable coverage, and proposition (1) holds.

(2) When $|T|=2$, only need to place one relay in anywhere of intersection of two vertexes' distance circles to ensure reliable coverage, proposition (1) holds.

(3) When $\left|T^{\prime}\right| \geq 3$, according to the nature of the group, $\forall T_{i}, T_{j} \in T^{\prime}, \exists e_{i, j} \in E^{\prime}, s_{i} \cap s_{j}$; let $d_{\min }=\min \left\{d T_{i}, T_{i} \in T^{\prime}\right\}$, if Error! Reference source not found., according to the properties of the equilateral triangle all distance circle of vertexes within the group are crossed by.

$$
\underset{T_{i} \in T,}{\cap} S_{j} \neq \varnothing
$$

If $\left|T^{\prime}\right|=k(k \geq 3)$, the proposition (1), (2) have been set up, namely

$$
\left\{\cap_{i=1}^{k} S_{j}\right\} \neq \varnothing
$$

Just need to deploy one relay at any point in the intersection of all vertexes's distance circles, we can ensure reliable coverage of these vertexes, the proposition is also true if we want to prove $|T|=s+1 \mid$.

Using contradiction, assume $\left|T^{\prime}\right|=s+1$, the original proposition is not true, that is, for all the $(s+1)$ vertexes in a group $g\left(T^{\prime}, e^{\prime}\right)$, at least need to deploy two relays to ensure their reliable coverage, these two relays are marked as R1 and R2.

Since in $g\left(T^{\prime}, e^{\prime}\right)$, even remove a vertex and the corresponding edge, it is still a group, denoted by $g *\left(T^{*}, e^{*}\right)$, without loss of generality, so let the vertex as $\left|T^{\prime}\right|=s+1,\left|T^{*}=T^{\prime} /\left\{T_{k}+1\right\}\right|=k$, so in $g *$ all vertexes just a relay to ensure the reliable coverage. We may assume that R1 provides reliable coverage for $k$ vertexes in $T^{*}$, there is intersection between these $s$ vertexes' distance circle $s_{1} \cap s_{2} \ldots \cap s_{k} \neq \varnothing$ Error! Reference source not found. Then the reliable coverage provided by R2 is divided into the following two situations:

(1) R2 provides reliable coverage for $T_{k}+1$ and other vertexes in group $g$ *, namely in $\left\{s_{i}, i=1,2, \ldots, k\right\}$ there is at least one $s_{j}(1 \leq j \leq k)$.

$$
g *\left\{\begin{array}{c}
s_{k+1} \cap s_{j} \neq \varnothing \\
\left\{s_{i}\right\} / s_{j} \cap s_{k+1} \neq \varnothing
\end{array}, 1 \leq i \leq k\right.
$$


Wherein formula (5) and $T$ ' is a group contradiction, so assumption does not hold.

(2) R2 only provides reliable coverage for $T s+1$,

$$
\left\{\begin{array}{c}
k \\
\cap s_{i} \\
i=1
\end{array}\right\} \cap s_{k+1} \neq \varnothing
$$

Formula (6) and $g$ ' is a group contradiction, the assumption is not true.

Therefore, when $\left|T^{\prime}\right|=s+1$ the proposition is also true

Based on the above three steps of the proof, according to mathematical induction, this theorem is proved. QED.

Since WIMAX network users generally distribute in places with high population density, the maximum distance between users is usually less than $100 \mathrm{~m}$, while the average user distance requirement is $100 \mathrm{~m} \sim 150 \mathrm{~m}$. Therefore, according to theorem 1 , in general WIMAX network, we conduct the least group partition according to the user adjacent diagram. After division, place a relay at any point in the intersection area of all users' distance circles in each group, to ensure reliable coverage of users in the regiment. Therefore, LORC problem can be transformed into minium clique partition problem of user adjacency graph.

As minimum clique partition problem (Minimum Clique Parti-tion problem, MCP) is NP-complete, Under normal circumstances, the design of MCP approximation algorithm is conducted in exceptional figure for the high complexity and time limitation, for example Unit Disk Graph (UDG) and penny graph. In this paper's studied LORC problem, the distance requirement of users is far greater than 1 and different from each other, therefore the distance circle figure is not diagram UDG; Because users distribute randomly in the circle and the circles will intersect, so it not Penny Graph either, so the existing MCP algorithm can not solve the LORC problem. Based on the user's neighbor information and user location information, the paper designs minimum clique partition heuristic algorithm.

\section{A. MAXDCP Heuristic Algorithm}

The basic idea is to divide a group that contains the most vertexes from the user adjacency graph, and removes the regiment from the adjacency graph, and then in the rest of the adjacency graph continue to divide groups according to the greedy rule, until every vertex in the user adjacency graph is included in a group.

Finding "group that contains the most vertexes" is the problem of find the maximum clique, it is NP-complete, usually finding for the largest group is difficult. Given that the more the user neighbors are, the more vertexes in the group, so start from the maximum vertex $\mathrm{v} \mathrm{m} \mathrm{a} \mathrm{x}$ of user adjacency graph to find all the groups that contains it, then carve out the group $C Q_{\max }$ with most vertexes. In this way, until all the vertexes in user adjacency graph are included in a group. The clique partition algorithm based on the maximum degree of vertex is described as below; Figure 2 shows all steps of MAXDCP. 


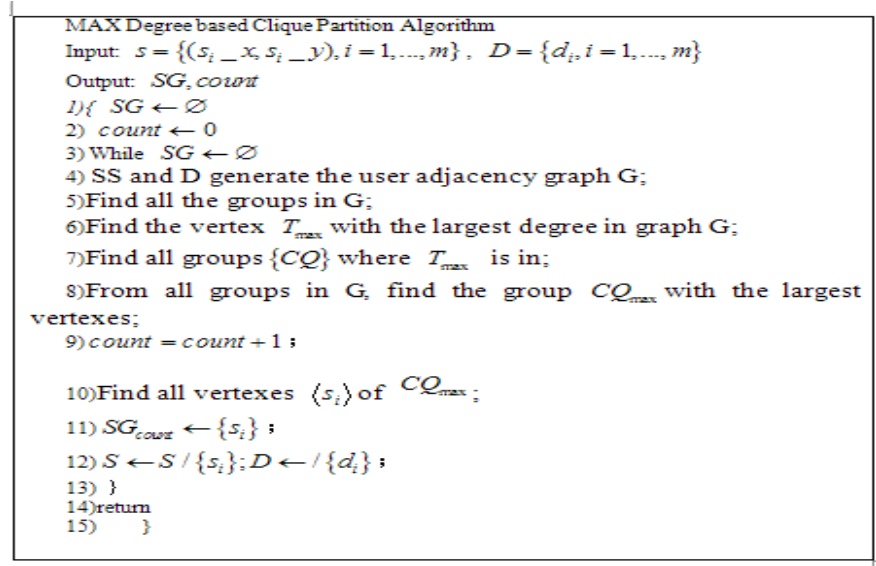

Figure 2. MAXDCP Algorithm

Since in practice, the base station location is known, so there are two possible methods about the process on the base station: The first method is to deploy relays in the case that the base station does not exist, when the relay deployment is complete, each user at least covered by one relay reliable coverage. Doing so, for users whose distance to the base station is smaller than the required distance, they could not only be covered by reliable relay coverage, but also can be covered by the base station's reliable relay coverage. These users can randomly select base station or a relay to transmit data. The second method firstly takes a base station with known location as a deployed relay and removes the users who have been covered by base station's reliable coverage from the user set, and then execute algorithm to handle the rest of the user. The second method will first known location of a base station has been deployed as repeaters and base stations have been reliable coverage of the collection is removed from the current user out, and then execute algorithms handle the rest of the user. Thus, in MAXDCP algorithm design, we take the first method for the base station.

Figure 3 (a) is a distance circle of user and its corresponding vertex, Fig 3 (b) is user adjacent graph, and Fig 3 (c) is a simple example of MAXDCP algorithm. As figure 3 (c) shows, MAXDCP starts from the maximum degree vertex (the 4th vertex) in the adjacency graph to search the group with the most vertexes to divide. However, since the divided group has the most vertexes in the current adjacency graph, all the edges which are connected with the vertex in the group are removed from the adjacency graph, So if the group is in the center of the adjacency graph, the maximum group division will break down other groups in the adjacency graph, so that the number of groups increase, increasing the scale of the problem, which is not conducive to minimizing the number of relay. In order to improve quality, we further consider the user position information in the area, propose algorithm GEOCP (GEO graphic information-based Clique Partition algorithm).

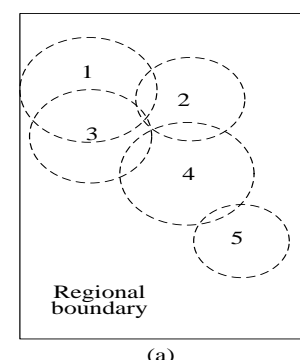

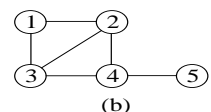

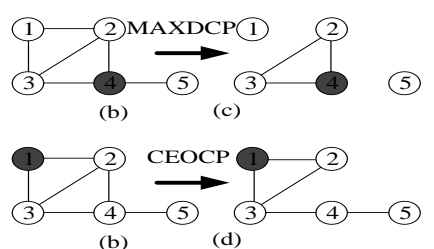

Figure 3. User Adjacency Graph and Algorithm's Clique Partition Example Based on MAXDCP, GEOCP 


\section{B. GEOCP Heuristic Algorithm}

The basic idea is, to identify the vertex where the boundary user locates from the adjacency graph, remove the maximum group which contains this boundary vertex from the adjacency graph, then in the rest of the adjacency graph continue to divide groups according to the greedy rule, until every vertex in the user adjacency graph is included in a group. Advantages to proceed from the boundary vertex to divide groups in the user adjacency graph are: each clique partition will not affect other groups' structure, there for it will not increase the number of groups in the adjacent figure. GEOCP algorithm is described in Figure 4:

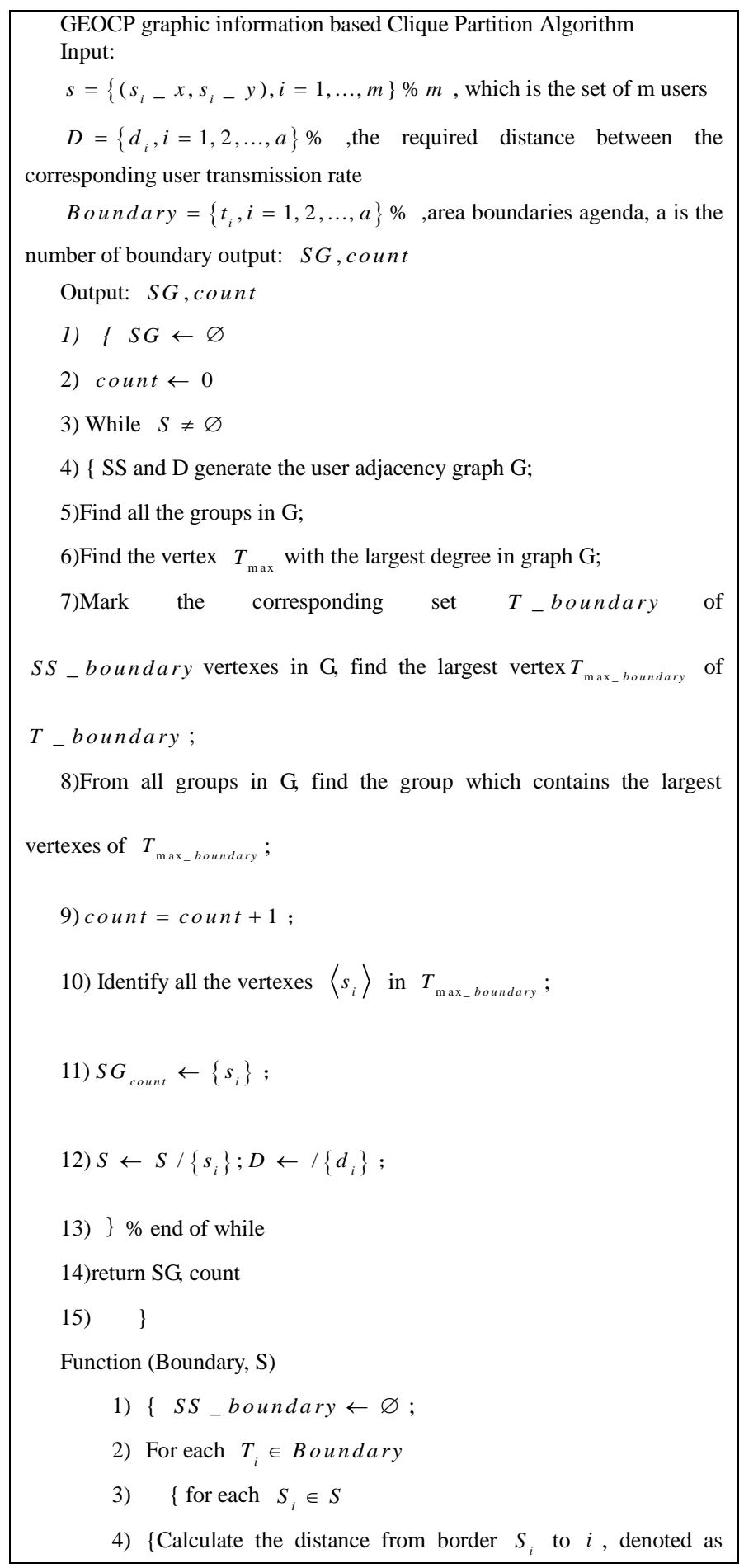




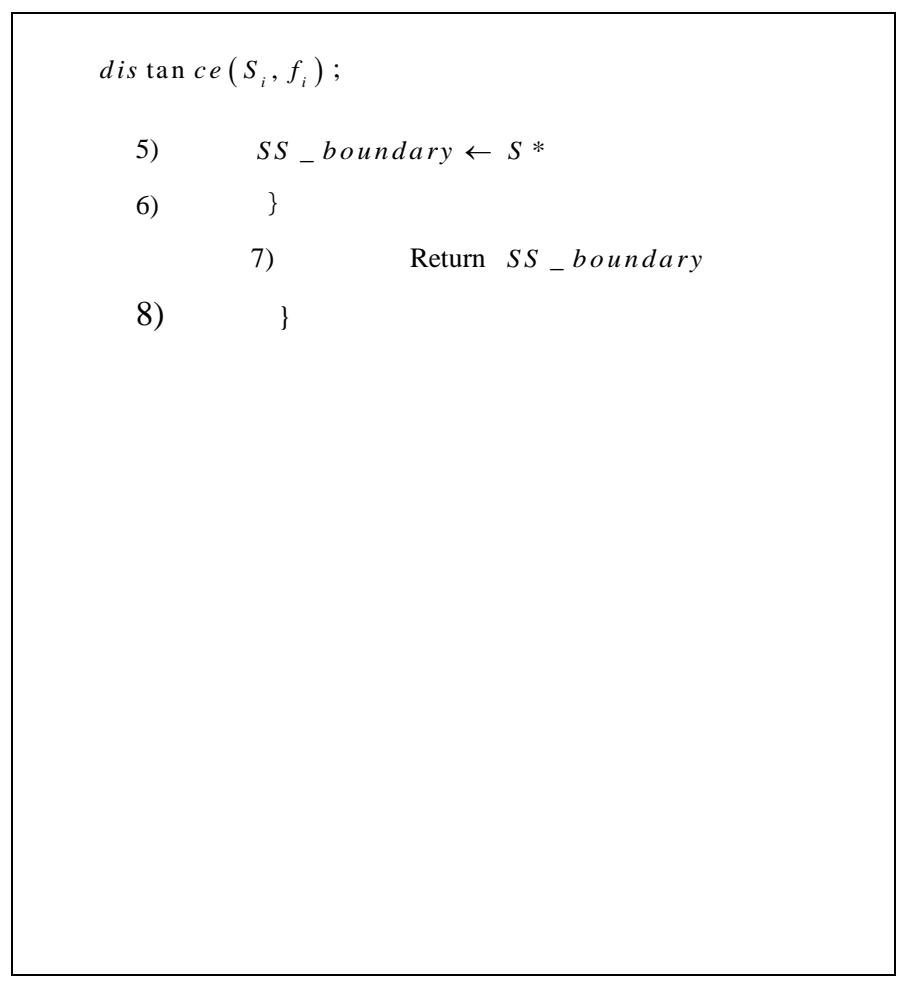

Figure 4. GEOCP Algorithm

\section{Simulation and Analysis}

This section compares MAXDCP, GEOCP, MIS and HS's effect through simulation. Assuming in a $1200 \mathrm{~m} \times 1200 \mathrm{~m}$ square area, one base station locates in the center of this area, the user are randomly distributed in the region, the user required distance is selected randomly within $[100,150]$. Simulation is conducted in a PC with the Intel Pentium Dual-Core $2.5 \mathrm{GHz}$ CPU, 32-bit Windows operating systems, the simulation tool is MATLAB 2010a.

\section{A. Network Topology}

Simulate that 50 users are randomly distributed in the region, based on WIMAX Grid network topology for relay deployment of MAXDCP algorithm and GEOCP algorithm. Respectively make MAXDCP algorithm and GEOCP algorithm deploy relay to ensure reliable coverage of the user, and then make MUST algorithm deploy the relay, so to ensure relay with reliable coverage can connect with the base station, Figure 5 shows the deployment of the relay based on different algorithms.

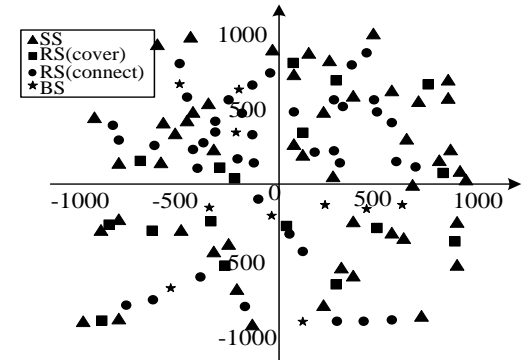

(a)MAXDCP+MUS

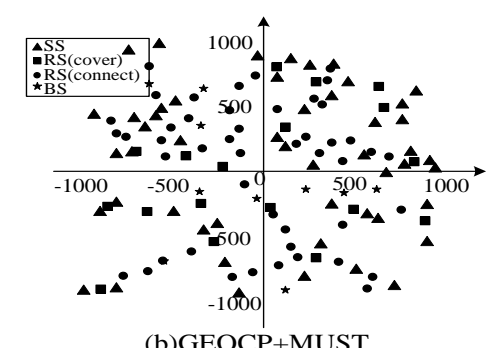

(b)GEOCP+MUST

Figure 5. Relay Deployment with a $1200 \mathrm{~m} \times 1200 \mathrm{~m}$ Area, One Base Station and 50 Users 
Among them, the 21 deployment coverage relays, 83 connected relays, MAXDCP + MUST totally deploy 104 relays. As every time MAXDCP selects the user group with the largest degree, i,e with maximum number of neighbors to conduct division, So if this user's group just in the area where users are dense, the division of the largest group will lead to other groups' decomposition, so need to deploy more relay.

Figure 5 (b) based on the GEOCP algorithm. Among them, 20 deployment coverage relays, 62 connected relays, GEOCP + MUST totally deploys 82 relays. As GEOCP combines user's location information and MAXDCP idea on the user partition, ensure that each division will not cause other user groups divide into smaller, more numerous groups, reducing the number of deployment relay s.

\section{B. Comparison of the Number of Deployed Relays}

To measure the effect of MAXDCP algorithm and GEOCP algorithm, we compare them with deployed relay's number of existing algorithms MIS and HS, and compare the total running time. Field is set to a $1200 \mathrm{~m} \times 1200 \mathrm{~m}$ square region, one base station in the center of the region, the number of users increase from 52 to 500 with increment of 52 , the user distance requirement is taken randomly from $[100,155]$, data shown are the average results after the algorithm performs 20 times .

As figure 6 (a) shows, in order to ensure the user's reliable coverage, the number of deployed relays of MAXDCP algorithm is reduced by about $35 \%$ than MIS; the number of deployed relays of GEOCP algorithm is reduced by about $56 \%$ than MIS, reduced by approximately $21 \%$ than HS, and the rate of increase in the number of relay of GEOCP is significantly lower than the other three algorithms. This is because MAXDCP and GEOCP only deploy the relays to users within a group, and within a group just one relay can ensure reliable coverage for all users in the group; the algorithm guarantees to minimize the number of divided groups so to minimize the number of deployed relays. Although MIS also generates user adjacency graph, but it selects one of the 6 vertexes around the user which with the minimum distance requirement to place relay, if the minimum distance of relay on one vertex covers other users, then place one relay . In the area with dense users, MIS algorithm will result in one user covered by several relays, the extent of this redundancy will increase as the user density becomes large. The HS algorithm is based on the minimum Hitting set, it deploy relays in intersection of distance circles. In this way, the more intensive the user, the more intersections of distance circles, which not only cause coverage redundancy on users but also increases algorithm's time complexity.

MAXDCP, GEOCP, MIS and HS deploy relays to ensure reliable coverage for users to solve the sub-problem LORC of DARP problem, then based on the location of reliable relay coverage, just need to deploy least relay to ensure multi-hop connectivity with the base station, to make MUST achieve connection between relay and the base station. The basic idea of MUST algorithm is base station as the root, relay provided reliable coverage as the leaf node to generate European Steiner tree with limited length. Wherein, it relays with reliable coverage generate from the above four different algorithms. Figure 6 (b) shows comparison about the number of relays which ensure communication, as shown in the figure. The number of connectivity relays of MAXDCP + MUST is less about $8 \%$ than MIS + MUST, the number of connectivity relays of GEOCP + MU ST is less 17 percent than MIS+MUST and it is closed to the number of HS + MUST relay. This is because when the user distribution is the same, the number of reliable coverage relay which as the leaf nodes determines the number of relays that MUST requires, But when the distance between the relays that provide reliable coverage is far, it needs more relays to ensure connectivity between these relays and between relays and the base station.

Figure 6 (c) shows comparison of the total number of all the relays that MAXDCP, GEOCP, MIS, and the HS's DARP problem needs. The total number of relays based on 
MAXDCP decrease 23.9\% compared with MIS, the total number of relays based on GEOCP reduces by $35 \%$ than MIS, decreases $18.5 \%$ compared with HS.

Figure 6 (d) shows the machine time that algorithm MAXDCP, GEOCP, MIS and HS respectively needs to solve the problem DARP. We note that, compared with the other three algorithms, the running time of HS + MUST algorithm apparently increases as the user density increases, This is because the HS bases on the minimum Hitting set, it selects deployment relays at the intersection of the distance circle of all vertexes, the user density increases, resulting in the number of intersections increases sharply, making the size of calculation of HS increases sharply. In contrast, GEOCP + MUST achieve the relay deployment which is similar to that of HS + MUST, by a lower run-time.

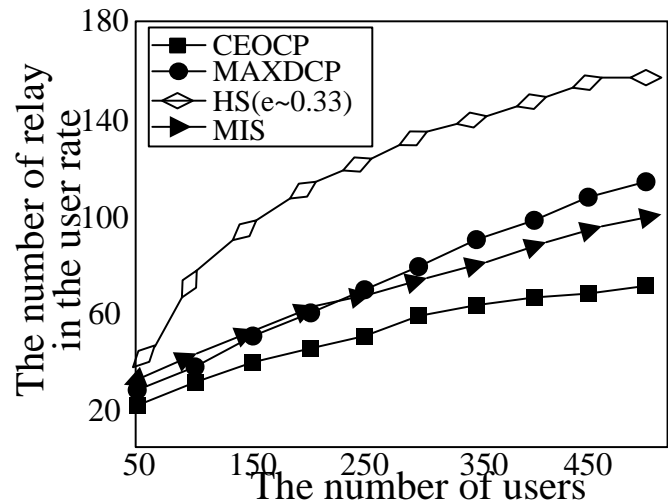

(a)Ensure the user the number of relay in the cover

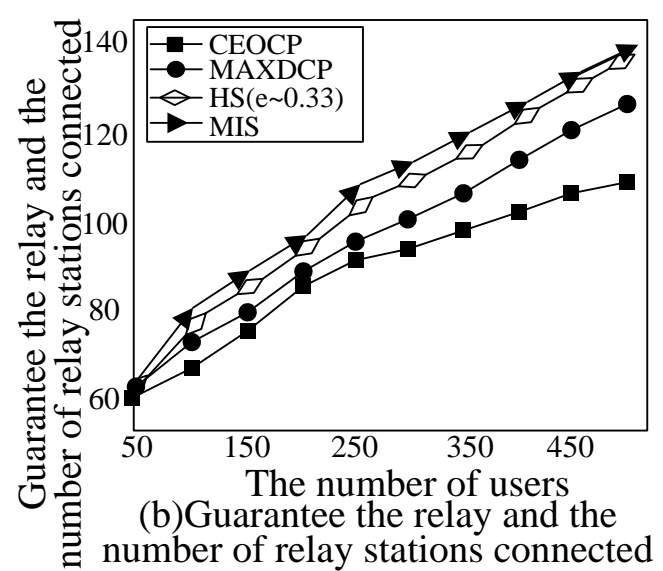

(d)The elapsed time

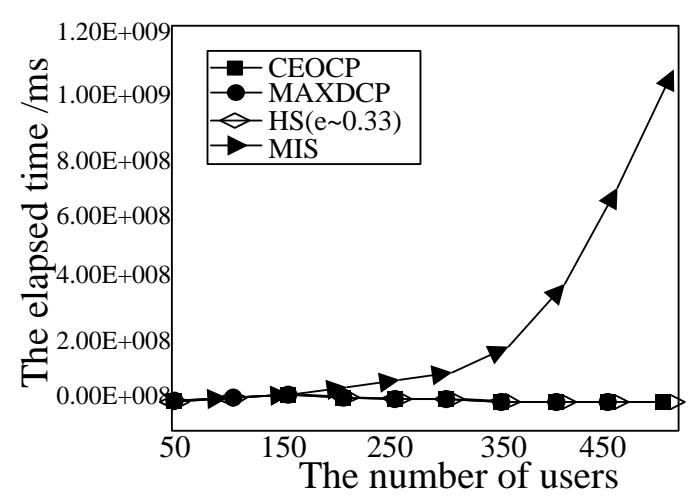

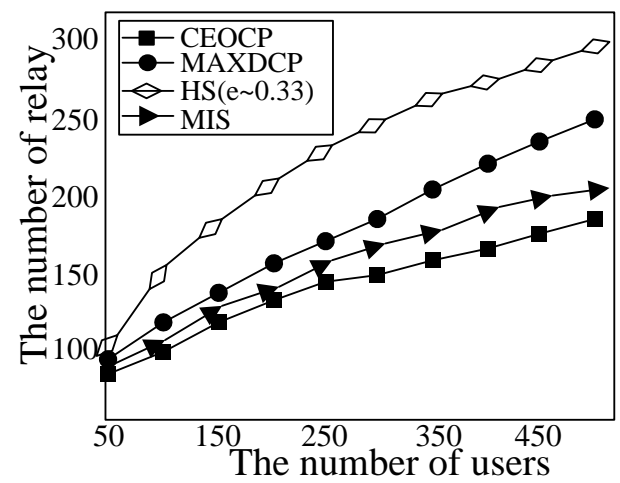

(c)The total number of relay Figure 6. Contrast of the Number of Deployed Relays between the
Proposed Algorithm and Algorithm MIS and HS $(1200 \mathrm{~m} \times 1200 \mathrm{~m}$ area)

From the above simulation results it can be concluded, MAXDCP and GEOCP algorithm which based on group division can achieve a known-distance relay deployment with less time and lower time complexity, ensures the required transmission rate for access link, reducing the cost of networking.

\section{Conclusion}

This paper studies the WIMAX Grid network's least relay deployment to meet the user's reliable coverage LORC, firstly proves that LORC issue is equivalent to solving problems of minimum clique partition in the user adjacency graph, the problem is NP-complete. Then, based on user neighbor information and user location information, proposes greedy heuristics MAXDCP and GEOCP. The simulation results show that: the proposed algorithm guarantees the user's distance requirements, compared with existing 
algorithms; it has advantages such as with fewer deployed relays and lower time complexity.

\section{Acknowledgment}

This research work is supported by the Education Department of Henan province science and technology research project (14A520046).

\section{References}

[1] M. J. Mirza and N. Anjum, "Association of Moving Objects Across Visual Sensor Networks", Journal of Multimedia, vol. 7, no. 1, (2012), pp. 2-8.

[2] Y. D. Lin, S. L. Tsao, S. L. Chang, et. al., "Design issues and experimental studies of wireless LAN grid," IEEE Wireless Communications, vol.17, no.2, (2010), pp. 32-40.

[3] J. D. Camp and E. W. Knightly, "The IEEE 802.1 Is Extended Service Set Grid Networking Standard," IEEE Communications Magazine, (2008) August, pp. 120-126.

[4] R. C. Carrano, L. C. S. Magalhaes, D. C. Muchaluat Saade, et. al., "IEEE 802.1 Is multihop MAC: a tutorial," IEEE Communications Surveys \& Tutorials, vol.13, no. 1, (2011), pp. 52-67.

[5] P. H. Pathak, R. Dutta, "A survey of network design problems and joint design approaches in wireless grid networks," IEEE Communications Surveys \& Tutorials, vol. 13, no .3, (2011), pp. 396-428.

[6] H. Zhai, "QoS Support over UWB Grid Networks", in proceedings of WCNC (2008), pp. 2283-2288.

[7] R. D. Taranto, H. Yomo, P. Popovski, et. al., "Cognitive Grid Network Under Interference from Primary User," Wireless Personal Communications, vol. 45, no. 3, (2013), pp. 385-401.

[8] K.H. Chi, Y. C. Shih, H. H. Liu, et. al., "Fast handoff in secure IEEE 802.11s grid networks," IEEE Transactions on Vehicular Technology, vol. 60, no. 1, (2010), pp. 219-232.

[9] T. Liu and W. Liao, "Interference-Aware QoS Routing for Multi-Rate Multi-Radio Multi-Channel IEEE 802.11 Wireless Grid Networks,' IEEE Transactions on Wireless Comm, vol. 8, no. 1, (2009) January, pp. 166-175.

[10] R. Hou, K. Lui, F. Baker, et. al., "Hop-by-hop routing in wireless grid networks with bandwidth Guarantees", IEEE Transactions on Mobile Computing, vol. 11, no. 2, (2011), pp. 264-277.

[11] S. L. Wu, C. Y. Lin, Y. C. Tseng, et. al., "A new multi-channel MAC protocol with on-demand channel assignment for multihop mobile Ad hoc networks," in Proc of ISPAN,Washington DC, IEEE Computer Society, (2000) ,pp. 232-237.

[12] Y. Geng, J. Chen and K. Pahlavan, "Motion detection using RF signals for the first responder in emergency operations", A PHASER project, 2013 IEEE 24nd International Symposium on Personal Indoor and Mobile Radio Communications (PIMRC), (2013) September, London,Britain.

[13] S. Li, Y. Geng, J. He and K. Pahlavan, "Analysis of Three-dimensional Maximum Likelihood Algorithm for Capsule Endoscopy Localization, 2012 5th International Conference on Biomedical Engineering and Informatics (BMEI)", (2012) October, pp. 721-725, Chongqing, China.

[14] M. Li and Y. Feng, "Design and Implementation of a Hybrid Channel-Assignment Protocol for a Multi-Interface Wireless Grid Network," IEEE Trans on Vehicular Technology, vol. 59, no. 6, (2010) July, pp. 2986-2997.

[15] L. Song,T. Zhang, X. Zeng, et. al., "Fair Bandwidth Allocation In Multi-radio Cognitive Wireless Grid Networks," in International Conference on WCSP, vol. 21-23, (2010) October, pp. 1-6.

[16] J. He, W. G. L. Bai, et. al., "Theoretic Analysis of IEEE 802.11 Rate Adaptation Algorithm SampleRate", IEEE Communications Letters, vol. 15, no. 5, (2011) May, pp. 524-526.

[17] L. Zhihan, L. Feng, S. Feng and H. Li, "Extending Touch-less Interaction on Vision Based Wearable Device." Virtual Reality (VR), 2015 iEEE. IEEE, (2015).

[18] M. Zhang, L. V. Zhihan, X. Zhang, G. Chen, and K. Zhang, "Research and Application of the 3D Virtual Community Based on WEBVR and RIA." Computer and Information Science 2, no. 1, (2009), pp. 84. 
[19] T. Su, L. V. Zhihan, S. Gao, X. Li and L. V. Haibin, "3D seabed, 3D modeling and visualization platform for the seabed", In Multimedia and Expo Workshops (ICMEW), 2014 IEEE International Conference on, (2014), pp. 1-6.

[20] S. Kim, L. Verma, S. Choi, et. al., "Collision-Aware Rate Adaptation in multi-rate WLANs, Design and implementation,” Computer Networks, vol. 54, (2010), pp.3011-3030.

\section{Author}

Ma Shaohui, was born in 1971. She is currently an associate professor in the Henan Mechanic and Electrical Engineering College. Her research interest is mainly in the area of Computer Software, Mechanical and Electrical Integration. She has published several research papers in scholarly journals in the above research areas. 